\title{
Etude de la flore de levure du Saint-Nectaire
}

\author{
par \\ Jeanne VERGEADE, J. GUIRAUD, J. P. LARPENT \\ et P. GALZY \\ Laboratoire de Microbiologie \\ Sciences et Techniques des Industries Alimentaires \\ Université des Sciences et Techniques du Languedoc \\ 34060 Montpellier Cedex \\ et \\ Laboratoire de Microbiologie \\ Centre de Biologie et Physiologie Végétales \\ 4 ct 6, rue Ledru- 63000 Clermont-Ferrand
}

Il semble que les levures jouent un rôle important dans la fabrication et l'affinage du fromage. Des travaux ont été consacrés à la flore de levure du Roquefort par Devoyod et al. [1, 2, 3] et par Galzin et al. [4], ainsi qu'à celle d'autres fromages bleus par Hartley et Jezeski [5], Maxa et Jicinsky [6], Proks et al. [7], Kanauchi et al. [8]. La flore de levure a été étudiée également dans le fromage de Limburger par El Erian [9], dans le Camembert par Lenoir et Auberger $[10,11]$, dans le Saint-Paulin par Lenoir et Ducastelle [12], dans le Vacherin par Sozzi [13], et dans le Cantal par Devoyod et al. [14].

Le mémoire présenté ici est consacré à l'étude de la flore de levure du Saint-Nectaire.

\section{MATERIEL ET METHODES}

\section{A) Techniques de prélèvement}

Les expériences ont été réalisées à partir de fromages provenant de trois fabricants différents : une fabrication laitière L (laiterie Riches Monts) et deux fabrications fermières A et B.

Les prélèvements ont été effectués pour des fromages d'une même fabrication à divers stades de l'affinage (jusqu'à 8 semaines). Chaque fromage a fait l'objet d'un prélèvement au niveau de la croûte sur une profondeur de $2 \mathrm{~mm}$ à l'aide d'un scalpel stérile et au niveau de la pâte à $4 \mathrm{~cm}$ de profondeur à l'aide d'une sonde stérile. 
Les échantillons ( $5 \mathrm{~g}$ environ) ont été broyés manuellement dans un mortier stérile avec $45 \mathrm{ml}$ de tryptone- $\mathrm{NaCl}$. Des dilutions logarithmiques ont été ensuite réalisées dans un milieu tryptone- $\mathrm{NaCl}$ Citrate ; $0,1 \mathrm{ml}$ de chaque dilution ont été étalés sur des boîtes de Pétri contenant du milieu Potato-Dextrose-Agar acidifié à pH 3,5 par l'acide tartrique. Les boîtes ont été incubées à $25^{\circ} \mathrm{C}$. Après $72 \mathrm{~h}$, un dénombrement a été réalisé en utilisant les boîtes contenant entre 20 et 200 colonies. Les colonies des boîtes correspondant aux plus faibles dilutions ont été prélevées ainsi que celles présentant un aspect particulier sur les autres boîtes. Après purification sur milieu liquide de Sabouraud-Chloramphénicol les souches ont été conservées sur milieu Yeast Extract-Glucose gélosé.

\section{B) Techniques d'identification}

Nous avons utilisé les techniques d'identification préconisées par Lodder [15].

\section{RESULTATS EXPERIMENTAUX}

\section{A) Etude taxonomique}

Nous avons étudié 156 souches obtenues chacune à partir d'une colonie.

Le groupe le plus important correspond à Debaryomyces hansenii Lodder et Kreger Van Rij : 134 souches. La plupart de ces souches (100) sporulent sur milieu V 8 en donnant au préalable une conjugaison isogamique ou une fusion entre cellule mère et bourgeon. Une seule spore se forme dans chaque asque, Les quelques souches non sporulées auraient pu être classées dans l'espèce Torulopsis candida (Saito) Lodder mais il nous a paru préférable d'étudier ensemble ces 134 souches qui présentent de nombreux points communs. D'ailleurs, ce point de vue est admis par Lodder qui place dans les Debaryomyces des souches non sporulées.

La plupart de ces souches (123) correspondent à la définition de l'espèce prévue par Lodder. La description donnée par Lodder prévoit une grande variabilité de l'espèce pour les caractères suivants : fermentation du glucose, du saccharose, du galactose et du maltose : + ou - ; présence ou non d'un voile ; assimilation du lactose, de l'inuline et de l'érythritol + ou - . En fonction de cette variabilité, nous pouvons-grouper ces 123 souches en 21 types biologiques (tab. 1). Les 11 autres souches correspondent, pour l'essentiel, à la description type de Debaryomyces hansenii mais en différent par au moins un caractère. Elles peuvent être groupées en 10 types biologiques en fonction de la variabilité admise pour l'espèce (tab. 2). Le tableau 3 indique les différences entre ces souches et la description type de l'espèce. Il apparaît que la variabilité génétique de Debaryomyces 
TABLEAU 1. - Caractéristiques des divers types biologiques de Debaryomyces hansenii

Le tableau représente les caractères variables selon Lodder

\begin{tabular}{|c|c|c|c|c|c|c|c|c|c|c|}
\hline \multirow{2}{*}{$\begin{array}{l}\mathrm{N}^{\circ} \\
\text { du type } \\
\text { biologi- } \\
\text { que }\end{array}$} & \multirow{2}{*}{$\begin{array}{l}\text { Nombre } \\
\text { de } \\
\text { souches } \\
\text { isolées }\end{array}$} & \multirow{2}{*}{$\begin{array}{c}\text { Sporula- } \\
\text { tion } \\
\text { V } 8\end{array}$} & \multicolumn{4}{|c|}{ Fermentation } & \multicolumn{3}{|c|}{ Assimilation } & \multirow{2}{*}{$\begin{array}{l}\text { Voile } \\
\text { sur } \\
\text { milieu } \\
\text { liquide }\end{array}$} \\
\hline & & & glucose & saccharose & galactose & maltose & lactose & inuline & érythritol & \\
\hline 1 & $\Lambda$ & & & & & & & & & \\
\hline $\begin{array}{l}1 \\
2\end{array}$ & $\begin{array}{l}4 \\
3\end{array}$ & $\begin{array}{l}+ \\
+\end{array}$ & $\begin{array}{l}+ \\
+\end{array}$ & + & + & - & + & - & - & + \\
\hline 3 & 10 & 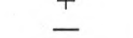 & $\begin{array}{l}+ \\
+\end{array}$ & $\begin{array}{l}+ \\
+\end{array}$ & $\begin{array}{l}+ \\
+\end{array}$ & - & + & - & $\overline{1}$ & - \\
\hline 4 & 2 & + & + & + & + & - & + & - & + & + \\
\hline 5 & 2 & + & + & + & + & - & $\bar{t}$ & $\bar{t}$ & + & + \\
\hline 6 & 2 & + & + & + & + & + & $\begin{array}{l}+ \\
+\end{array}$ & \pm & - & + \\
\hline 7 & 1 & + & + & + & + & + & $\begin{array}{l}+ \\
+\end{array}$ & - & - & + \\
\hline 8 & 3 & - & + & + & + & + & + & - & - & - \\
\hline 9 & 1 & - & + & + & + & + & $\begin{array}{l}+ \\
+\end{array}$ & $\bar{t}$ & + & + \\
\hline 10 & 2 & + & + & + & - & + & + & + & + & + \\
\hline 11 & 1 & + & + & + & - & + & $\stackrel{+}{-}$ & $\overline{-}$ & - & + \\
\hline 12 & 2 & - & + & + & - & + & $\bar{t}$ & - & - & + \\
\hline 13 & 21 & + & - & - & - & - & + & - & + & + \\
\hline 14 & 2 & + & - & - & - & - & + & $\bar{t}$ & - & - \\
\hline 15 & 2 & + & - & - & - & - & + & \pm & - & - \\
\hline 16 & 2 & + & + & - & - & - & + & - & + & - \\
\hline 17 & 18 & + & + & + & - & - & + & - & - & - \\
\hline 18 & 38 & - & + & + & - & - & + & - & - & + \\
\hline 19 & $\cdot 7$ & + & + & + & - & - & + & $\overline{+}$ & - & - \\
\hline 20 & 10 & + & + & + & - & - & + & - & - & - \\
\hline 21 & 1 & - & + & + & - & - & - & - & + & + \\
\hline & & & & & & & & & & \\
\hline
\end{tabular}


TABLEAU 2. - Caractéristique des types biologiques de Debaryomyces hansenii différant par au minimum un caractère de la description de l'espèce type selon Lodder

Le tableau représente les caractères variables selon Lodder

\begin{tabular}{|c|c|c|c|c|c|c|c|c|c|c|}
\hline \multirow{2}{*}{$\begin{array}{c}\mathrm{N}^{\circ} \\
\text { du type } \\
\text { biologi- } \\
\text { que }\end{array}$} & \multirow{2}{*}{$\begin{array}{c}\text { Nombre } \\
\text { de }\end{array}$} & \multirow{2}{*}{$\begin{array}{l}\text { Sporula- } \\
\text { tion } \\
\text { V } 8\end{array}$} & \multicolumn{4}{|c|}{ Fermentation } & \multicolumn{3}{|c|}{ Assimilation } & \multirow{2}{*}{$\begin{array}{l}\text { Voile } \\
\text { sur } \\
\text { milieu } \\
\text { liquide }\end{array}$} \\
\hline & & & glucose & saccharose & galactose & maltose & lactose & inuline & érythritol & \\
\hline $\begin{array}{l}22 \\
23 \\
24 \\
25 \\
26 \\
27 \\
28 \\
29 \\
30 \\
31\end{array}$ & $\begin{array}{l}1 \\
1 \\
1 \\
1 \\
2 \\
1 \\
1 \\
1 \\
1 \\
1\end{array}$ & $\begin{array}{l}+ \\
+ \\
+ \\
+ \\
+ \\
+ \\
+ \\
+ \\
+\end{array}$ & $\begin{array}{l}+ \\
+ \\
+ \\
+ \\
+ \\
+ \\
+ \\
+ \\
+ \\
+\end{array}$ & $\begin{array}{l}+ \\
+ \\
+ \\
+ \\
+ \\
+ \\
+ \\
+ \\
+ \\
+\end{array}$ & $\begin{array}{l}+ \\
+ \\
+ \\
+ \\
= \\
= \\
= \\
=\end{array}$ & $\begin{array}{l} \pm \\
\pm \\
= \\
= \\
= \\
= \\
=\end{array}$ & $\begin{array}{l}+ \\
+ \\
+ \\
+ \\
+ \\
+ \\
+ \\
+ \\
+ \\
+\end{array}$ & $\begin{array}{l}\overline{-} \\
\overline{-} \\
\overline{-} \\
\overline{+} \\
\pm \\
=\end{array}$ & $\begin{array}{l}- \\
= \\
= \\
- \\
\pm \\
= \\
=\end{array}$ & $\begin{array}{l}+ \\
+ \\
+ \\
+ \\
+ \\
+ \\
= \\
= \\
-\end{array}$ \\
\hline
\end{tabular}




\section{TABLEAU 3}

Différences entre les types biologiques 22 à 31 et l'espèce type Debaryomyces hansenii selon Lodder

\begin{tabular}{|c|c|}
\hline $\begin{array}{l}\text { Types } \\
\text { biologiques }\end{array}$ & $\begin{array}{c}\text { Différence avec l'espèce type Debaryomyces hansenii } \\
\text { selon Lodder }\end{array}$ \\
\hline $\begin{array}{l}22 \\
23 \\
24 \\
25 \\
26 \\
27 \\
28 \\
29 \\
30 \\
31\end{array}$ & $\begin{array}{l}\text { Assimilation du mannitol négative } \\
\text { Assimilation du maltose, cellobiose, } \mathrm{L} \text { arabinose négative } \\
\text { Assimilation du raffinose négative } \\
\text { Assimilation du } \mathrm{L} \text { arabinose négative } \\
\text { Assimilation du maltose, cellobiose, } \mathrm{L} \text { arabinose négative } \\
\text { Assimilation du mannitol et raffinose négative } \\
\text { Assimilation du saccharose et mannitol négative. } \\
\text { Assimilation du galactose, } \mathrm{L} \text { arabinose et xylose négative } \\
\text { Assimilation du } \mathrm{L} \text { arabinose et xylose négative } \\
\text { Assimilation du } \mathrm{L} \text { arabinose négative }\end{array}$ \\
\hline
\end{tabular}

hansenii est considérable puisque les tests de fermentation peuvent être + ou - pour les substrats suivants : glucose, galactose, saccharose, maltose. De même, les tests d'assimilation sont variables (+ ou -) pour les substrats carbonés suivants : galactose, L sorbose, saccharose, maltose, cellobiose, lactose, mélibiose, raffinose, inuline, D xylose, $\mathrm{L}$ et $\mathrm{D}$ arabinose, $\mathrm{D}$ ribose, $\mathrm{L}$ rhamnose, érythritol, galactitol, D mannitol, acide citrique et pour les composés azotés suivants : nitrite de sodium et créatine.

Un deuxième groupe est constitué par l'espèce Torulopsis sphaerica Van der Walt (15 souches). Il faut y ajouter une souche sporulée que nous avons rattachée, de ce fait, à l'espèce Kluyveromyces lactis Van der Walt.

Les autres espèces trouvées ne l'ont été qu'exceptionnellement :

Torulopsis versatilis Lodder et Krejer Van Rij (1 souche).

Candida intermedia Langeron et Guerra (2 souches).

Candida kéfyr Van Uden et Buckley (1 souche).

Candida scottii Diddens et Lodder (1 souche).

Cryptococcus flavus Phaff et Fell (1 souche).

Le tableau $\mathrm{n}^{\circ} 4$ indique le nombre de souches de chaque espèce pour un fromage laitier et deux fromages fermiers. Nous avons étudié environ 50 souches par fromage, ces souches prélevées en partie sur la croûte et en partie au centre de la pâte. Les histogrammes de la figure 1 indiquent en outre dans les trois fromages, la fréquence des divers types biologiques de Debaryomyces hansenii. Il semble que la flore des fromages fermiers soit un peu plus variée que celle du fromage laitier. Cependant, compte tenu de la rareté de certaines espèces et de certains types biologiques, il est peu probable que leur présence soit importante. 


\section{TABLEAU 4}

Répartition des levures isolées en fonction de la zone de prélèvement et du type de fromage

\begin{tabular}{|c|c|c|c|c|c|c|}
\hline \multirow{2}{*}{ Espèce } & \multicolumn{2}{|c|}{$\begin{array}{c}\text { Fromage } \\
\text { laitier } \\
\text { L }\end{array}$} & \multicolumn{2}{|c|}{$\begin{array}{c}\text { Fromage } \\
\text { fermier } \\
\text { A }\end{array}$} & \multicolumn{2}{|c|}{$\begin{array}{c}\text { Fromage } \\
\text { fermier } \\
\text { B }\end{array}$} \\
\hline & pâte & croûte & pâte & croûte & pâte & croûte \\
\hline Kluyveromyces lactis & 0 & 0 & 1 & 0 & 0 & 0 \\
\hline Torulopsis sphaerica & 1 & 2 & 5 & 0 & 6 & 1 \\
\hline Debaryomyces hansenii & 26 & 21 & 26 & 21 & 23 & 18 \\
\hline Torulopsis versatilis & 0 & 0 & 0 & 0 & 1 & 0 \\
\hline Candida intermedia & 0 & 0 & 0 & 0 & 2 & 0 \\
\hline Candida kefyr & 1 & 0 & 0 & 0 & 0 & 0 \\
\hline Candida scottii & 0 & 0 & 1 & 0 & 0 & 0 \\
\hline Cryptococcus flavus & 0 & 0 & 1 & 0 & 0 & 0 \\
\hline
\end{tabular}

Fromage L

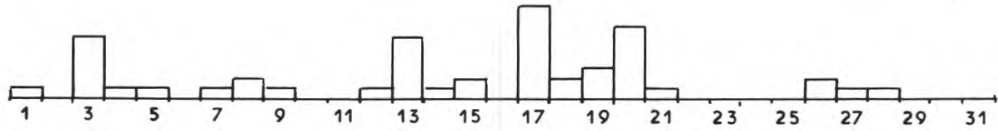

Fromage A
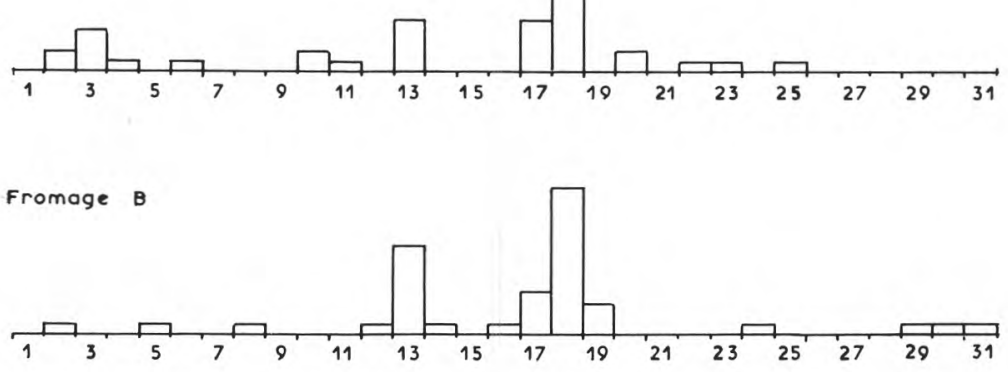

fig. 1

Histogramme de la fréquence des différents types biologiques de Debaryomyces hansenii isolés dans les trois fromages.

Nous ne donnons pas de description détaillée des souches qui sont conformes, dans l'ensemble, aux définitions données par Lodder pour les diverses espèces. Cependant, nous annexons à ce mémoire les photographies des cellules des diverses espèces (fig. 2). 


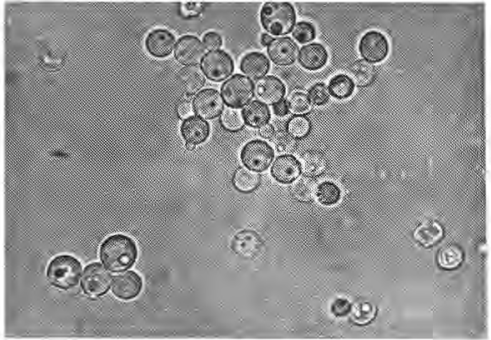

A

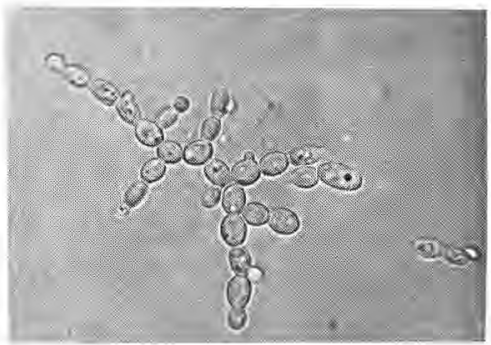

C

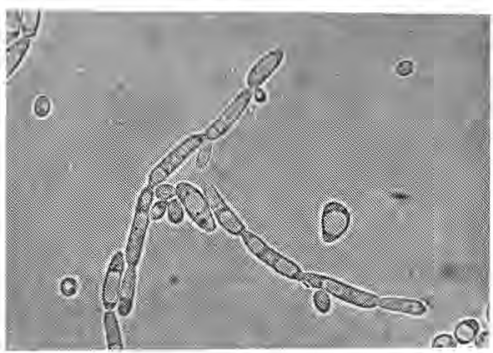

$\mathrm{E}$

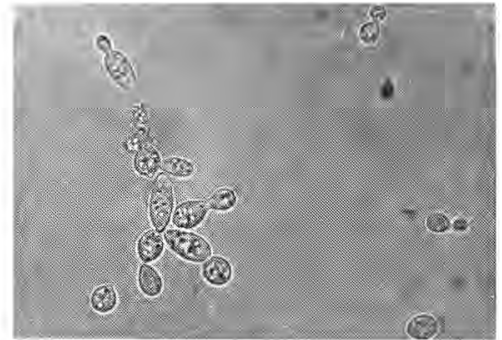

G

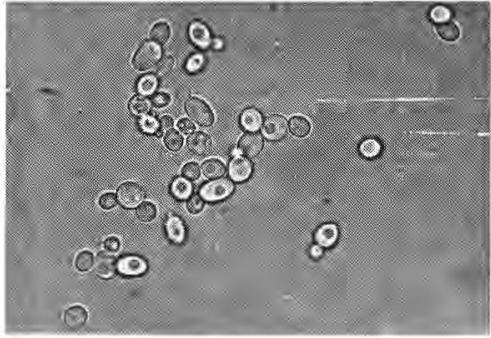

B

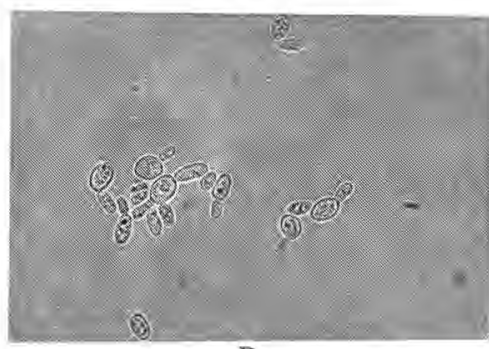

D

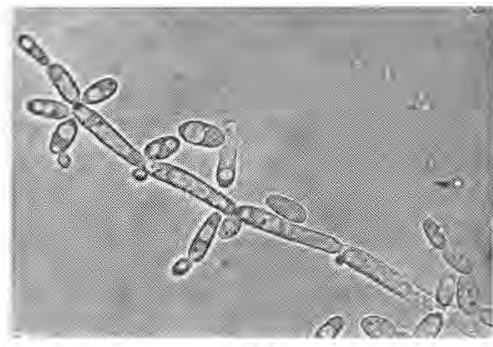

$\mathrm{F}$

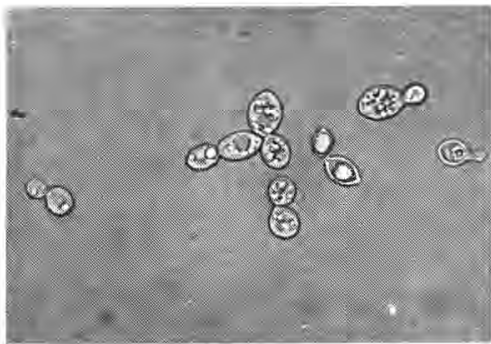

$\mathrm{H}$

fig. 2

Aspect microscopique des cellules des différentes espèces isolées (milieu yeast extract glucose gélosé ; grossissement $\times 180$ ).
A - Debaryomyces hansenii
C - Torulopsis sphaerica
$\mathrm{B}$ - Kluyveromyces lactis
$\mathrm{E}$ - Candida intermedia
$\mathrm{D}$ - Torulopsis versatilis
$\mathrm{G}$ - Candida scottii
F - Candida kefyr
$\mathrm{H}-$ Cryptococcus flavus 


\section{B) Etude quantitative de la flore}

La méthode utilisée pour le prélèvement des colonies dans les boîtes de Pétri après étalement permet d'estimer l'importance quantitative de chaque espèce. Les tableaux 5 à 7 indiquent, pour chaque fromage, les pourcentages relatifs des deux grands groupes (Debaryomyces hansenii et Torulopsis sphaerica) et des autres espèces groupées sous la rubrique divers. Nous indiquons aussi l'importance de la flore totale. Ces tableaux indiquent l'évolution de la flore au cours de la maturation entre la $1^{\text {re }}$ et la $8^{\mathrm{e}}$ semaine ; ils permettent de distinguer la situation dans la croûte et dans la pâte.

Le groupe Debaryomyces hansenii-Torulopsis candida est toujours dominant quel que soit le fromage.

Le groupe Kluyveromyces lactis-Torulopsis sphaerica est toujours présent au début de l'affinage. Il se trouve surtout dans la croûte du fromage laitier alors qu'il est également abondant dans la pâte des fromages fermiers. Cependant, après la $4^{\mathrm{e}}$ semaine, les deux espèces

TABLEAU 5

Evolution de la flore de levure au cours de l'affinage dans le fromage laitier L

\begin{tabular}{|c|c|c|c|c|c|c|c|c|}
\hline Date en semaines & \multicolumn{2}{|c|}{1 à 2} & \multicolumn{2}{|c|}{3 à 4} & \multicolumn{2}{|c|}{5 à 6} & \multicolumn{2}{|c|}{7 à 8} \\
\hline Zone de prélèvement & pâte & croûte & pâte & croûte & pâte & croûte & pâte & croûte \\
\hline $\begin{array}{c}\text { p. } 100 \\
\text { Torulopsis } \\
\text { sphaerica } \\
- \\
\begin{array}{c}\text { Kluyveromyces } \\
\text { lactis }\end{array}\end{array}$ & 0 & 10 & 3 & 10 & 0 & 0 & 0 & 0 \\
\hline $\begin{array}{c}\text { p. } 100 \\
\text { Debaryomyces } \\
\text { hansenii }\end{array}$ & & & & & & & & \\
\hline $\begin{array}{c}\text { Torulopsis } \\
\text { candida }\end{array}$ & 100 & 90 & 77 & 90 & 100 & 100 & 100 & 100 \\
\hline $\begin{array}{l}\text { p. } 100 \\
\text { Divers }\end{array}$ & 0 & 0 & 20 & 0 & 0 & 0 & 0 & 0 \\
\hline $\begin{array}{c}\text { Flore totale } \\
\times 10^{6} \text { cellules } / \mathrm{g} \\
\text { de fromage }\end{array}$ & 0,01 & 100 & 0,08 & 200 & 0,07 & 1000 & 0,01 & 100 \\
\hline
\end{tabular}




\section{TABLEAU 6}

Evolution de la flore de levure au cours de l'affinage dans le fromage fermier A

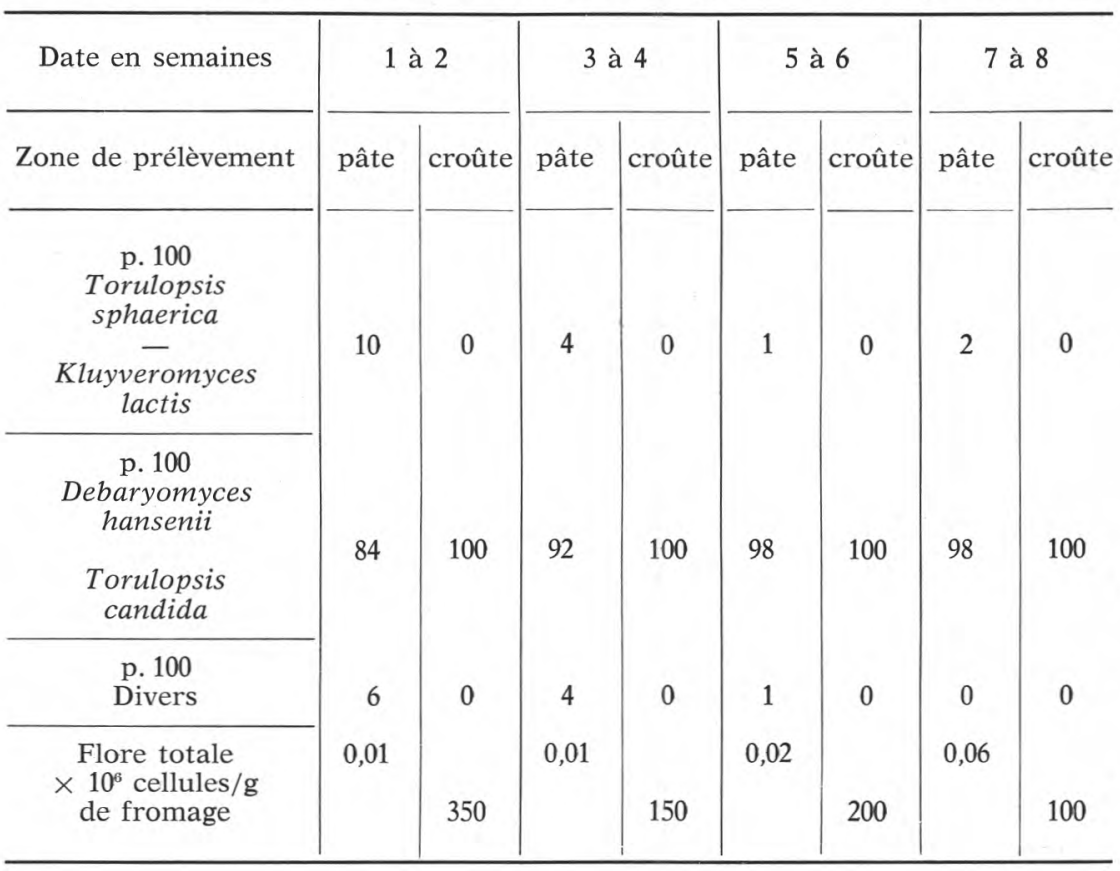

TABLEAU 7

Evolution de la flore de levure au cours de l'affinage dans le fromage fermier B

\begin{tabular}{|c|c|c|c|c|c|c|c|c|}
\hline Date en semaines & \multicolumn{2}{|c|}{1 à 2} & \multicolumn{2}{|c|}{3 à 4} & \multicolumn{2}{|c|}{5 à 6} & \multicolumn{2}{|c|}{7 à 8} \\
\hline Zone de prélèvement & pâte & croûte & pâte & croûte & pâte & croûte & pâte & croûte \\
\hline $\begin{array}{c}\text { p. } 100 \\
\text { Torulopsis } \\
\text { sphaerica } \\
- \\
\text { Kluyveromyces } \\
\text { lactis }\end{array}$ & 10 & 0 & 55 & 0 & 50 & 0 & 50 & 0 \\
\hline $\begin{array}{c}\text { p. } 100 \\
\begin{array}{c}\text { Debaryomyces } \\
\text { hansenii }\end{array}\end{array}$ & & & & & & & & \\
\hline $\begin{array}{c}\text { Torulopsis } \\
\text { candida }\end{array}$ & 85 & 100 & 45 & 100 & 50 & 100 & 50 & 100 \\
\hline $\begin{array}{l}\text { p. } 100 \\
\text { Divers }\end{array}$ & 5 & 0 & 0 & 0 & 0 & 0 & 0 & 0 \\
\hline $\begin{array}{c}\text { Flore totale } \\
\times 10^{6} \text { cellules } / \mathrm{g} \\
\text { de fromage }\end{array}$ & 0,4 & 400 & 0,1 & 1000 & 0,1 & 100 & 0,1 & 100 \\
\hline
\end{tabular}


disparaissent dans le fromage laitier et leur nombre diminue fortement dans l'un des deux fermiers. Au contraire, il prolifère dans le $2^{\mathrm{e}}$ fromage fermier et atteint 50 p. 100 de la flore de levure de la pâte en fin d'affinage. Il est probable que le développement des souches de ce groupe dans le fromage dépend beaucoup des conditions d'affinage.

Les autres espèces ne sont présentes qu'à titre exceptionnel.

\section{CONCLUSION}

Les levures sont nombreuses dans le fromage de Saint-Nectaire.

Le groupe Debaryomyces hansenii-Torulopsis candida est le plus abondant et le seul à être universellement répandu dans le SaintNectaire. C'est certainement, parmi les levures, le groupe qui joue le rôle essentiel dans la dégradation de l'acide lactique et la remontée du $\mathrm{pH}$, en relation avec l'action des champignons de la croûte.

Le groupe Kluyveromyces lactis-Torulopsis sphaerica présente de grandes variations et n'est pas toujours présent en fin d'affinage. Il est probable que ce groupe est sensible aux conditions de fabrication et d'affinage. Il est possible qu'il joue un grand rôle dans les variations de qualité organoleptique observées entre les divers fromages.

\section{Remerciements}

Nous remercions la Société Riches Monts pour l'aide qu'elle nous a apporté pour la réalisation de ce travait.

\section{R és u m é}

Les levures du fromage Saint-Nectaire appartiennent aux espèces Debaryomyces hansenii - Torulopsis candida et Kluyveromyces lactis Torulopsis sphaerica. La répartition des divers groupes varie selon le type de fabrication du fromage.

\section{S u m m a r y}

Yeasts of Saint-Nectaire cheese belong to species Debaryomyces hansenii-Torulopsis candida and Kluyveromyces lactis-Torulopsis sphaerica. Repartition of species depend of making-type of cheese. 


\section{Bibliographie}

[1] Devoyod (J. J.) et Bret (C.) (1966). - Evolution de la flore microbienne au cours de la fabrication et de l'affinage du fromage de Roquefort. XVII ${ }^{\mathrm{e}}$ Congrès International de Laiterie D, 585-594.

[2] Devoyod (J. J.), BRet (C.) et Auclatr (J.) (1968). - La flore microbienne du fromage de Roquefort. I. Son évolution au cours de la fabrication et de l'affinage. Le Lait, 479-480, 613-630.

[3] Devoyod (J.) et Sponem (D.) (1970). - La flore microbienne du fromage de Roquefort.. VI. Les levures. Le Lait, 498, 524-543.

[4] Galzin (M.), Galzy (P.) et Bret (G.) (1970). - Etude de la flore de levure dans le fromage de Roquefort. Le Lait, 491-492, 1-37.

[5] Hartley (C. B.) and Jezeski (J. J.) (1954). - The microflora of blue cheese slime. J. Dairy Sci., 37, 436.

[6] MaXa (V.) and Jicinsky (V.) (1956). - A study of some asporogenous yeasts in view of their application in the manufacture of Roquefort cheese. Int. Dairy Congr., 20, 464.

[7] Proks (J.), Dolezalek (J.) and PeCH (Z.) (1959). - A study of the coction of the yeasts of the genus Torulopsis on the formation of the methyl ketones in the cheese of Roquefort type. Int. Dairy Congr., 2, 345.

[8] Kanauchi (T.), Yoshioka (Y.) and Hamamoto (M.) (1962). - Microbial studies on blue veined cheese. I. Microflora of blue veined cheese in ripening period. Jap. J. Zootech. Sci., 32, 104.

[9] EL ERIAN (1969). - Bacteriological studies on Limburger cheese. MededLandbouwhoge-school, Wageningen, 12, 1.

[10] Lenorr (J.) (1962). - La flore microbienne du Camembert et son évolution au cours de la maturation. C.R. Ac. Agr., 48, 392-399.

[11] Lenoir (J.) et Auberger (1966). - Contribution à l'étude de la flore microbienne du fromage de type Camembert. C.R. XVII ${ }^{e}$ Cong. Int. Lait., D 595.

[12] Lenorr (J.) et Ducastelle (A.) (1965). - Contribution à l'étude de la flore microbienne du fromage de Saint-Paulin.

I. Son évolution au cours de la maturation. Le Lait, 447, 371-378.

II. Les espèces dominantes. Le Lait, 448, 509-518.

[13] Sozzi (T.) et SHEPERd (O.) (1972). - Evolution de la composition chimique et de la flore microbienne du fromage de Vacherin au cours de la maturation du lait. Le Lait, 52, 203-219.

[14] Devoyod (J. J.), Millet (L.) et Melcion (D.) (1974). - La flore microbienne du fromage de Cantal fabriqué à partir de lait cru. I. Techniques d'études et résultats préliminaires. Le Lait, 537, 397-407.

[15] Lodder (J.) (1970). - The yeast. North Holland Publishing Company, Amsterdam-London. 